\title{
Experimental Evaluation of Inventory-Based Discrete-Updating Market Maker for Intra-firm Prediction Market System Using VIPS
}

\author{
Hajime Mizuyama, Morio Ueda, Katsunobu Asada, and Yu Tagaya \\ Department of Mechanical Engineering and Science, \\ Kyoto University, Kyoto 606-8501, Japan \\ mizu@me.kyoto-u.ac.jp
}

\begin{abstract}
This paper develops an intra-firm prediction market system as a collective-knowledge-based forecasting tool for a company and evaluates its performance through laboratory experiments. The system uses the variableinterval prediction security (VIPS) as the prediction security to be traded in the market and is controlled by an original computerized market maker suitable for the security type. The market maker evaluates each unit of VIPS with a Gaussian price distribution and updates the distribution intermittently through an inventory-based updating logic according to the transactions in the market. Laboratory experiments are conducted with a virtual demand forecasting problem to study whether the system functions properly as a subjective forecasting tool. The experiments confirm that the system is capable of penalizing arbitrage actions and hence its performance is fairly stable. Further, the output price distribution can serve as an approximate forecast distribution.
\end{abstract}

Keywords: Collective knowledge, prediction markets, demand forecasting, information aggregation.

\section{Introduction}

To obtain an adequate demand forecast for a product in a future time period in the rapidly changing market environment, it is often insufficient or inappropriate to rely only on the information extracted from its historical sales data through a statistical method. Similar difficulties have also been encountered in other forecasting problems in a company, in recent years. Under the circumstances, one of the potentially valuable approaches to tackle the difficulties is to utilize the flexible forecasting capability of human agents, and which can be readily accomplished through a prediction market. The prediction market is a virtual futures market of a prediction security whose worth depends on the unknown realized value of a random variable of interest, and thus the market price of the security provides a dynamic forecast on the random variable reflecting the dispersed knowledge of the participants [1], [2], [3], [4]. Early industrial applications include [5], [6] and [7].

The prediction market is rapidly becoming popular, and now many companies are interested in testing it as an intra-firm forecasting tool. However, most applications 
reported up to now are either for obtaining only a point estimate of a certain variable with the vote-share-type prediction security or for achieving subjective probabilities of the occurrence of several mutually exclusive events with the winner-takes-all-type prediction security. Whereas, most forecasting problems in a company are those require a forecast distribution of a variable of interest. Thus, the authors have introduced a new type of prediction security called the variable-interval prediction security (VIPS) and an intra-firm prediction market system with a computerized market maker suitable for the security type [8].

An arbitral prediction interval regarding the variable of interest can be specified to VIPS, and each unit of which will pay off a prescribed amount of money, when the market is closed, if and only if the realized value of the variable is actually contained in the interval. During the market is open, the computerized market maker accepts every offer of buying or selling VIPS given by a participant at a price calculated according to a Gaussian price distribution. It is also required of the market maker to update the price distribution itself so that it can be regarded as a latest collective forecast of the participants. It is found that a simple weighted average updating logic is vulnerable to arbitrage actions [9]. Hence, the inventory-based updating logic and the book value constraint are further introduced in order to fix the vulnerability [10].

This paper is aimed at implementing the refined market maker into a pilot prediction market system and conducting laboratory experiments on the system to confirm that it can actually diminish the arbitrage actions and will function properly as a subjective forecasting tool in the real world. The remainder of the paper first presents the outline of the developed system, then discusses the experimental design, the experimental results and their implications, and finally concludes the paper.

\section{Intra-firm Prediction Market System}

\subsection{Outline of Pilot Market System}

The market system is aimed at providing a company with a continuous forecast distribution of, for example, the demand quantity $x$ of a certain product in a predetermined time period in the future. The system is supposed to be run for a certain time period with not so many participants who are potentially knowledgeable about the variable of interest $x$, for example, the salespeople of the company. The system provides a marketplace where the participants can buy and sell VIPS concerning $x$. The transactions are play-money-based and all with the market maker of the system. The play money used is referred to as the prediction dollars (P\$).

Each unit of VIPS pays off a fixed amount of prediction dollars after the market is closed, if and only if the realized value of $x$ is contained in the specified prediction interval. Each participant $k(=1,2, \ldots, K)$ is given a certain amount of prediction dollars as the initial endowment and is properly motivated to invest it on VIPS so as to increase her/his posterior wealth. In the following laboratory experiments, the initial endowment is $10000(\mathrm{P} \$)$ and the unitary payoff is $100(\mathrm{P} \$)$.

Each participant $k$ can hold plural units of VIPS but their prediction intervals must be identical. Thus, her/his VIPS holding position can be represented by $\left(a_{k}, b_{k}, v_{k}\right)$, where $\left[a_{k}, b_{k}\right]$ represents the prediction interval and $v_{k}$ denotes how many units. 
Further, the amount of prediction dollars at hand is denoted by $u_{k}$. Each participant $k$ can buy some additional VIPS from or sell some of the owned VIPS to the market maker easily by changing the position parameters $\left(a_{k}, b_{k}, v_{k}\right)$, as far as $u_{k}$ does not become negative. In the following laboratory experiments, the values of $\left(a_{k}, b_{k}, v_{k}\right)$ are bound to $[0,5000]$.

The market maker of the system has a Gaussian price density $N\left(\mu_{g}, \sigma_{g}^{2}\right)$, whose integral from $a_{k}$ to $b_{k}$ times 100 determines the unitary price of VIPS with interval $\left[a_{k}\right.$, $b_{k}$ ]. The price distribution is updated by the market maker intermittently every time an offer of buying or selling VIPS given by a participant has been processed.

\subsection{System Architecture and Its Implementation}

Simple client-server architecture is used for the pilot market system. The server part is built on a commercial database software and the client part is installed in PCs that are connected to the server machine through LAN. Hence, the entire system can be easily implemented in a typical intranet environment. The server part stores all the necessary information mainly in the asset profile table, the transaction history table and the price history table. The asset profile table contains the current values of $a_{k}, b_{k}, v_{k}$, and $u_{k}$ of each participant. Whereas the transaction history table is a stack of the records of these parameter values, etc. with a time stamp corresponding to every transaction. The price history table is a growing list of the values of $\mu_{g}$ and $\sigma_{g}$.

The client part for each participant is realized as a GUI-based system which communicates with the server part in the structured query language. It mainly provides three functions; current status visualization, position change simulation, and transaction realization. The current status visualization function first receives the current asset profile of the participant and the price history information from the server part. Then it visualizes the current shape of the price density distribution and her/his current VIPS holding position simultaneously on a same window, and displays the historical shapes of the price distribution under the window as a sequence of box and whisker plots. It also shows the contingent values of the posterior wealth as a bar chart. Further, by opening a separate window, she/he can consult a simple table showing the current VIPS holding positions of the other participants.

The position change simulation function allows the participant to perform simulations of changing her/his VIPS holding position on the GUI before actually changing it. The simulation can be easily done by dragging the square representing her/his VIPS holding position by a mouse in the main window. Then it shows the resultant contingent values of the posterior wealth after the position change as a separate bar chart. When the participant likes the resultant position, she/he can easily realize it by activating the transaction realization function with pushing a button. Then, the transaction realization function automatically updates the related values in the asset profile table, and adds a record of the updated position, the cost of the update, the parameter values of the corresponding price distribution, the time stamp, etc. to the transaction history table. It also updates the price distribution, and adds a record of the updated values of $\mu_{g}$ and $\sigma_{g}$ to the price history table. 


\subsection{Possible Arbitrage Actions and Their Prevention}

As described earlier, a simple weighted average price updating logic was found to be vulnerable to the following arbitrage actions [9]:

A. To escalate the price density around a certain prediction interval by buying many units of VIPS in the area, and then make a profit by soon selling the units at the boom price.

B. To lower the price density around a certain interval she/he actually wants by buying many units of VIPS away from the area, and then buy the interval she/he actually wants at the compressed price.

Thus, the pilot market system developed here diminishes the opportunities for these arbitrage actions by adopting an inventory-based updating logic called the LMSR-like and the book value constraint [10].

The LMSR-like updating logic first captures the inventory of VIPS owned by a participant $k$ as:

$$
s_{k}(x)=\left\{\begin{array}{cc}
v_{k} & \left(a_{k} \leq x \leq b_{k}\right) \\
0 & \text { otherwise }
\end{array}\right.
$$

and the whole inventory of VIPS in the market as $S(x)=s_{1}(x)+s_{2}(x)+\cdots+s_{K}(x)$.It then determines the values of the mean and variance of the price distribution by those values of the distribution of $\exp \{S(x) / \eta\}$, where $\eta$ is a parameter that controls the price updating sensitivity. This is called the LMSR-like because it can be regarded as a Gaussian approximation of the LMSR market maker proposed by [11].

Whereas the book value constraint works as follows. Each time a participant buys a set of VIPS, the price density at the moment is stored into the database as the book value information corresponding to the set. And when she/he offers to sell, at least a part of, the set later, the offer will be settled at the cheaper price between the corresponding book value and the current market value. When an offered position change has both a selling part and a buying part, the book value constraint has the market maker settle only the selling part first, and after updating the price density once, then settle the remaining buying part. The inventory-based updating logic has a preferable characteristic that when a participant sells a set of VIPS that she/he has just bought, the price distribution goes back to the shape before she/he bought the set. Hence, when combined with the book value constraint, it can diminish the arbitrage opportunities $\mathrm{A}$ and $\mathrm{B}$ even under the discrete updating frequency.

\section{Laboratory Experiments}

\subsection{Research Questions and Experimental Design}

The laboratory experiments are conducted in two experimental sessions. Each session consists of six experimental terms. The number of participants is nine in session 1 and seven in session 2. The participants are graduate or undergraduate students in different departments of Kyoto University. In each term, a virtual sales history of a 
product from January to September $\left(d_{1}, d_{2}, \ldots, d_{9}\right)$ is shown to the participants, and they are expected to speculate on the demand quantity $x=d_{12}$ to be realized in December. The virtual sales history data are generated by:

$$
d_{t}=\beta_{0}+\beta_{1} \cdot(t-6.5)+e \quad e \sim N\left(0,300^{2}\right)
$$

where the values of the parameters $\beta_{0}$ and $\beta_{1}$ are drawn from the uniform distributions [2250, 2750] and [-87.5, 100] respectively. This is formally a simple linear regression problem, but which is not revealed to the participants. In order to make the participants motivated towards the contest to maximize their posterior wealth, the participation fee to be actually paid to them for each term is set to be negatively proportional to the order of their posterior wealth.

The research questions considered in the experiments are whether the refined market maker successfully diminishes the arbitrage actions and whether the price distribution converges to an adequate forecast distribution. To answer the questions, the experimental design matrix shown in the left half of Table 1 is used. The sessions are treated as blocks, and each session is designed as a factorial design between two control factors Eta and Info. Eta represents the sensitivity parameter $\eta$ of the market maker and has three levels 300, 500 and 700. Info controls how information arrives and has two levels $\mathrm{S}$ and $\mathrm{D}$. When Info is $\mathrm{S}$, all the corresponding sales history information is shown to the participants at the beginning of the term and the term is continued for five minutes. Whereas if it is D, only the sales history from January to March is shown to them at starting the term, and when three minutes elapses that from April to June and when six minutes passes the rest of the information are disclosed respectively. In this case, the term lasts nine minutes in total.

Table 1. Experimental design matrix and experimental results

\begin{tabular}{c|c|c|c|c|c|c|c|c|c}
\hline Term & Session & Eta & Info & $P e$ & $D l t$ & $\mu_{g}$ & $\sigma_{g}$ & $C m$ & $C s$ \\
\hline 1 & 1 & 300 & $\mathrm{~S}$ & 2314 & 879 & 2374 & 321 & 0.07 & 0.37 \\
\hline 2 & 1 & 500 & $\mathrm{~S}$ & 2454 & 1096 & 2320 & 404 & 0.12 & 0.37 \\
\hline 3 & 1 & 700 & $\mathrm{~S}$ & 2120 & 1288 & 2278 & 671 & 0.12 & 0.52 \\
\hline 4 & 1 & 300 & $\mathrm{D}$ & 2773 & 1091 & 2855 & 324 & 0.08 & 0.30 \\
\hline 5 & 1 & 500 & $\mathrm{D}$ & 2432 & 1207 & 2659 & 611 & 0.19 & 0.51 \\
\hline 6 & 1 & 700 & $\mathrm{D}$ & 2337 & 850 & 2474 & 629 & 0.16 & 0.74 \\
\hline 7 & 2 & 300 & $\mathrm{~S}$ & 2200 & 787 & 2143 & 337 & 0.07 & 0.43 \\
\hline 8 & 2 & 500 & $\mathrm{~S}$ & 2845 & 1116 & 2626 & 604 & 0.20 & 0.54 \\
\hline 9 & 2 & 700 & $\mathrm{~S}$ & 2163 & 1027 & 2367 & 835 & 0.20 & 0.81 \\
\hline 10 & 2 & 300 & $\mathrm{D}$ & 2863 & 770 & 2488 & 227 & 0.49 & 0.30 \\
\hline 11 & 2 & 500 & $\mathrm{D}$ & 2227 & 1067 & 2360 & 580 & 0.12 & 0.54 \\
\hline 12 & 2 & 700 & $\mathrm{D}$ & 2099 & 621 & 2396 & 684 & 0.48 & 1.10 \\
\hline
\end{tabular}

\subsection{Experimental Results and Their Implications}

In the right half of Table 1, together with the point estimate $P e$ and the half-width of the $95 \%$ confidence interval Dlt achieved by linear regression, the parameter values of the final price distribution are shown. Further, $C m$ and $C s$ in the table denote the 
following measures of the appropriateness of the obtained mean and standard deviation relative to the point estimate and confidence interval:

$$
\begin{gathered}
C m=\left|\mu_{g}-P e\right| / D l t \\
C s=\sigma_{g} / D l t
\end{gathered}
$$

where $\mathrm{Cm}$ is a smaller-the-better measure, and $C s$ should take a value around 0.5 since $\mu_{g} \pm 2 \sigma_{g}$ covers approximately $95 \%$ of the distribution. Tables 2 and 3 show the results of analysis of variance applied to $\mathrm{Cm}$ and $\mathrm{Cs}$ respectively.

Table 2. ANOVA table of $\mathrm{Cm}$

\begin{tabular}{l|ccccc}
\hline & $\begin{array}{c}\text { Degrees of } \\
\text { freedom }\end{array}$ & $\begin{array}{c}\text { Sum of } \\
\text { squares }\end{array}$ & Mean square & F value & P value \\
\hline Eta & 2 & 0.015 & 0.007 & 0.468 & 0.645 \\
Info & 1 & 0.045 & 0.045 & 2.825 & 0.137 \\
Session & 1 & 0.056 & 0.056 & 3.499 & 0.104 \\
\hline Residuals & 7 & 0.112 & 0.016 & & \\
\hline
\end{tabular}

Table 3. ANOVA table of $C s$

\begin{tabular}{l|ccccc}
\hline & $\begin{array}{c}\text { Degrees of } \\
\text { freedom }\end{array}$ & $\begin{array}{c}\text { Sum of } \\
\text { squares }\end{array}$ & Mean square & F value & P value \\
\hline Eta & 2 & 0.418 & 0.209 & 12.471 & 0.005 \\
Info & 1 & 0.017 & 0.017 & 0.996 & 0.352 \\
Session & 1 & 0.071 & 0.071 & 4.254 & 0.078 \\
\hline Residuals & 7 & 0.117 & 0.017 & & \\
\hline
\end{tabular}

In most cases, the absolute bias of the obtained mean from the corresponding point estimate seems acceptable considering that the system is a subjective forecasting tool. It is also confirmed that any noticeably high biases, for example, those in terms 10 and 12 , can be attributed to the superficial curvature trend in the corresponding virtual sales history. Since it is not announced that the data is created by a simple linear model, capturing the curvature trend is not problematic for a subjective forecasting tool. The average magnitude of the obtained standard deviation also seems adequate. Thus, the output price distribution can serve as an approximate forecast distribution.

Table 3 shows that the standard deviation is significantly affected by Eta. Therefore, in order to further refine the output forecast distribution, the value of $\eta$ should be appropriately chosen from this aspect. In the current experimental setting, its desirable value seems to be around 500. How to fine-tune the sensitivity parameter in general setting is an important future research topic. It is also noted that the significance of the effect of Info is rejected on both $\mathrm{Cm}$ and $\mathrm{Cs}$. This implies that the newly introduced book value constraint does not seriously decrease the sensitivity of the market system to dynamically arriving information.

Next, it is studied how the price distribution changes along the transactions. In most cases, the process followed a smooth convergence to a certain distribution. It is also observed that, when Info is D, the arrival of additional information can change 

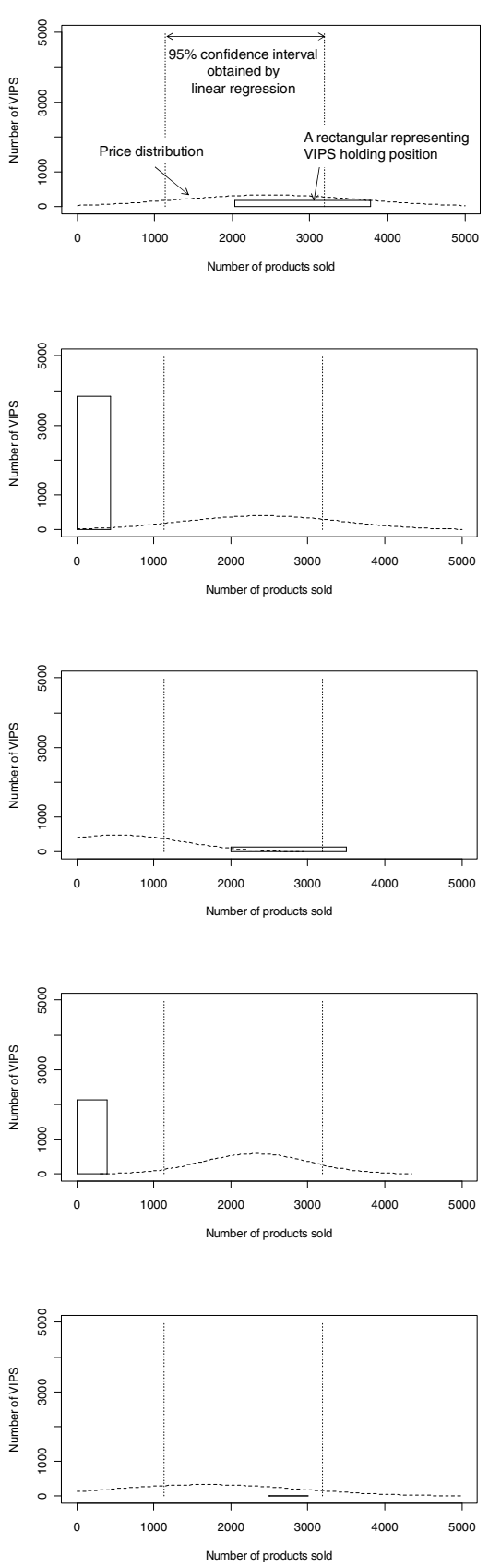

Fig. 1. How participant 5 changes his VIPS holding position in term 9; from the top figure to the bottom one the target distribution and accelerate the convergence to it, but the partial process between information arrivals looks similar to the process formed when Info is S. Thus, in those cases, the system does not seem to suffer from the arbitrage actions and successfully captures the provided information rapidly at the beginning and then gradually.

However, there are two exceptional cases, terms 9 and 11. In these cases, the price distribution became quire instable in the middle of the process. Investigating the transaction history table reveals that, in these terms, a few participants tried to take the arbitrage actions. For example, Fig. 1 shows how participant 5 changed his VIPS holding position in term 9. It is obvious from this figure that his second and fourth transactions were intended to take the arbitrage actions $\mathrm{A}$ and/or B. It is also observed that, on the contrary to his intension, these actions have shrunken his asset, after all into a tiny rectangular shown in the bottom of Fig. 1.That is, in this case, and in the other similar cases as well, the troublesome efforts towards arbitrage have been successfully punished and will not be continued. It is also confirmed that the disturbing transaction gave the other participants a chance to buy VIPS in an appropriate area at a very cheap price. The chance was soon taken by a shrewd participant and thus the price distribution soon came back into an appropriate shape. This is why the troublesome efforts towards arbitrage did not give a serious damage to the final price distribution.

One potential problem revealed by the observation above is that more than two participants as a team can still cheat the system. Although this may not be critical in the case of intra-company usage of the system, how to tackle the problem is an interesting future research topic. 


\section{Conclusions}

This paper developed an intra-firm prediction market system as a collectiveknowledge-based forecasting tool for a company and evaluated its performance through laboratory experiments with a virtual demand forecasting problem, which is essentially a linear regression problem. The experiments confirmed that the system is capable of penalizing the troublesome arbitrage actions and hence its performance will be fairly stable. It is also observed that the output price distribution can serve as an approximate forecast distribution. In order to further refine the output forecast distribution, how to fine-tune the sensitivity parameter in general setting is an important future research topic. Further, though it may not be critical for intracompany usage of the system, more than two participants as a team can still cheat the system. Thus, how to tackle the problem is also an interesting future research topic. Finally, field experiments will be required to put the developed market system into practical use.

Acknowledgments. This research is partially supported by the Ministry of Education, Science, Sports and Culture, Grant-in-Aid for Scientific Research (B) 20310087.

\section{References}

1. Plott, C.R.: Markets as Information Gathering Tools. Southern Economic Journal 67, 1-15 (2000)

2. Pennock, D.M., Lawrence, S., Giles, C.L., Nielsen, F.A.: The Real Power of Artificial Markets. Science 291, 987-988 (2001)

3. Wolfers, J., Zitzewitz, E.: Prediction Markets. Journal of Economic Perspectives 18, 107126 (2004)

4. Tziralis, G., Tatsiopoulos, I.: Prediction Markets: An Extended Literature Review. Journal of Prediction Markets 1, 75-91 (2007)

5. Ortner, G.: Forecasting Markets - An industrial Application Part I. A Working Paper in Dept. of Managerial Economics and Industrial Organization at TU Vienna (1997)

6. Ortner, G.: Forecasting Markets - An industrial Application Part II. A Working Paper in Dept. of Managerial Economics and Industrial Organization at TU Vienna (1998)

7. Chen, K., Plott, C.R.: Information Aggregation Mechanisms: Concept, Design and Implementation for a Sales Forecasting Problem. California Institute of Technology. Social Science Working Paper \#1131 (2002)

8. Mizuyama, H., Kamada, E.: A Prediction Market System for Aggregating Dispersed Tacit Knowledge into a Continuous Forecasted Demand Distribution. In: Olhager, J., Persson, F. (eds.) Advances in Production Management Systems, pp. 197-204. Springer, Heidelberg (2007)

9. Ueda, M., Mizuyama, H., Asada, K., Tagaya, Y.: Laboratory Experiments of Demand Forecasting Process through Intra-Firm Prediction Market System Using VIPS. In: Proceedings of 9th APIEMS Conference (2008)

10. Mizuyama, H.: A Prediction Market System Using VIPS for Collective-Knowledge-Based Demand Forecasting. In: Proceedings of the 20th International Conference on Production Research (2009) (to appear)

11. Hanson, R.: Combinatorial Information Market Design. Information Systems Frontiers 5, 107-119 (2003) 